\title{
Fréchet Kernel for Trajectory Data Analysis
}

\author{
Koh Takeuchi \\ takeuchi@i.kyoto-u.ac.jp \\ Kyoto University \\ Kyoto, Japan \\ Keisuke Fujii \\ Ken Yoda \\ Nagoya University \\ Nagoya, Japan
}

\author{
Masaaki Imaizumi \\ The University of Tokyo \\ Tokyo, Japan
}
Masakazu Ishihata
NTT
Kyoto, Japan

\author{
Shunsuke Kanda \\ Yasuo Tabei \\ RIKEN AIP \\ Tokyo, Japan
}
Takuya Maekawa
Osaka University
Osaka, Japan

\begin{abstract}
Trajectory analysis has been a central problem in applications of location tracking systems. Recently, the (discrete) Fréchet distance becomes a popular approach for measuring the similarity of two trajectories because of its high feature extraction capability. Despite its importance, the Fréchet distance has several limitations: (i) sensitive to noise as a trade-off for its high feature extraction capability; and (ii) it cannot be incorporated into machine learning frameworks due to its non-smooth functions. To address these problems, we propose the Fréchet kernel (FRK), which is associated with a smoothed Fréchet distance using a combination of two approximation techniques. FRK can adaptively acquire appropriate extraction capability from trajectories while retaining robustness to noise. Theoretically, we find that FRK has a positive definite property, hence FRK can be incorporated into the kernel method. We also provide an efficient algorithm to calculate FRK. Experimentally, FRK outperforms other methods, including other kernel methods and neural networks, in various noisy real-data classification tasks.
\end{abstract}

\section{CCS CONCEPTS}

-Information systems $\rightarrow$ Spatial-temporal systems; Data mining; Spatial-temporal systems; Data mining; Geographic information systems; • Computing methodologies $\rightarrow$ Machine learning approaches; Kernel methods.

\section{KEYWORDS}

datasets, neural networks, gaze detection, text tagging

\section{ACM Reference Format:}

Koh Takeuchi, Masaaki Imaizumi, Shunsuke Kanda, Yasuo Tabei, Keisuke Fujii, Ken Yoda, Masakazu Ishihata, and Takuya Maekawa. 2021. Fréchet Kernel for Trajectory Data Analysis. In 29th International Conference on Advances in Geographic Information Systems (SIGSPATIAL '21), November 2-5, 2021, Beijing, China. ACM, New York, NY, USA, 4 pages. https://doi.org/10.1145/ 3474717.3483949

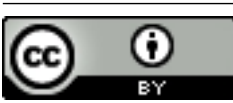

This work is licensed under a Creative Commons Attribution International 4.0 License. SIGSPATIAL '21, November 2-5, 2021, Beijing, China

(C) 2021 Copyright held by the owner/author(s).

ACM ISBN 978-1-4503-8664-7/21/11.

https://doi.org/10.1145/3474717.3483949

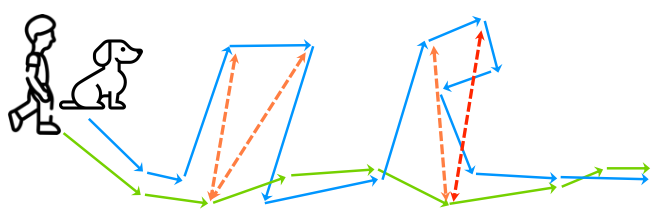

Figure 1: Illustration of the (discrete) Fréchet distance. Distance between the two trajectories is measured by the discrepancy between the most distant points (red dashed line). Other distant points (orange dashed lines) are ignored.

\section{INTRODUCTION}

Recent advancements in location tracking systems have enabled us to observe the trajectory data of various moving objects, such as airplanes, taxis, bicycles, pedestrians, sport players, and animals, and numerous applications utilizes these systems [32]. These trajectories have been applied to into various applications including trajectory classifications [14, 21, 23, 25, 33] and predictions $[7,12,16,17,29]$. For example, ecologists have utilized animal trajectories to understand survival strategies of animals and their relationships with natural environments. City officers have attempted to understand traffic situations from vehicle trajectories and decide route guidance for easing urban traffic congestion.

The (discrete) Fréchet distance [1,9] is a popular approach for measuring the similarity between trajectories. The Fréchet distance measures a gap between the two most distant points in a pair of trajectories given the best alignment of each point in the trajectories. It can be linked to the length of a lead needed to walk with a dog (see Fig. 1). The idea of using the most distant point in the best alignment enables us to extract trajectory features that consider both spatial locations and temporal order. Due to its remarkable feature extraction capacity, this technique has been successfully applied to a wide range of applications and demonstrated better performance $[3,4,24,26]$. This feature extraction capability is a unique feature of the Fréchet distance and is the opposite of another distance function (e.g., dynamic time warping distance (DTW) [2]) that smooths trajectories.

Despite its popularity, the Fréchet distance has critical problems in practice. First, it is very sensitive to noise, as a trade-off for its advantage in feature extraction. Because this distance evaluates only the two most distant points in a single alignment, if noise is added to those points, the distance may vary greatly. This limitation makes it impossible to use the Fréchet distance for noisy real data 
analysis such as the back-and-forth locations that can be recorded by inaccurate GPS sensors or from moving objects that frequently changes their movements and destinations. To deal with situations, filtering noisy points out from a trajectory has often taken but tends to eliminate characteristic properties of trajectories. Second, the Fréchet distance is inapplicable to commonly-used machine learning frameworks, such as kernel methods. Because the operation of choosing the most distant points under the best alignment is not mathematically differentiable, it is difficult to incorporate these distances into frameworks that strongly require smoothness (e.g. positive definite property).

To overcome these problems, we propose the Fréchet kernel (FRK) to be used in the general kernel framework for trajectories, based on an extended version of the Fréchet distance. Our FRK uses two smoothing techniques, namely the soft-min and smooth-min approximations, to eliminate the non-smooth factors of these original distances such as the selection of distant points and alignments. Moreover, we provide a computationally effective algorithm for FRK that can calculate outputs of this kernel with long trajectories, based on a probabilistic approximation approach. We conducted intensive trajectory classification experiments from five publicly available datasets consisting of various types of moving objects including automobiles, taxes, bicycles, pedestrians, sports players, and animals in the wild. We evaluated the performances of support vector machines (SVM) using FRK against that of baseline methods, including SVM with other existing kernels and end-to-end style recurrent neural networks (RNNs). Experimental results show that our methods achieve considerable performance improvements on the classification problems.

\section{PRELIMINARY EQUATIONS}

Let $p, q \in \mathbb{N}$ be lengths, and $m \in \mathbb{N}$ be a dimension of an observation point of trajectories. We consider two discrete trajectories, $X=$ $\left(x_{1}, \ldots, x_{p}\right) \in \mathbb{R}^{m \times p}$ and $Y=\left(y_{1}, \ldots, y_{q}\right) \in \mathbb{R}^{m \times q}$. To define their similarity, we define an alignment $A$ as a subset of a family of index pairs $\{(i, j) \in\{1,2, \ldots, p\} \times\{1,2, \ldots, q\}\}$ such that (i) $(1,1) \in A$ and $(p, q) \in A$ always hold; and (ii) if $(i, j) \in A$ holds, $A$ contains one of $(i+1, j),(i, j+1)$, or $(i+1, j+1)$. Let $\mathcal{A}$ be a set of all possible alignments $A$. The discrete Fréchet distance [8] can be defined as:

$$
D_{\text {Frechet }}(X, Y):=\min _{A \in \mathcal{A}} \max _{(i, j) \in A} d\left(x_{i}, y_{j}\right),
$$

where $d: \mathbb{R}^{m} \times \mathbb{R}^{m} \rightarrow \mathbb{R}$ is a distance between two vectors, such as the Euclid distance. The Fréchet distance seeks an alignment $A$ for two trajectories $X$ and $Y$ that minimizes the maximum distance of paired locations $\left(x_{i}, y_{j}\right)$. This formulation (1) can be efficiently computed by dynamic programming (DP) in $O(p q)$ time.

There are several approximation methods for max and min operators for vectors $z=\left(z_{1}, \ldots, z_{J}\right) \in \mathbb{R}^{J}$.

Soft-min approximation [6]: This approach approximates the minimum value with a parameter $\gamma>0$ as: $\exp \left(-\min _{j=1, \ldots, J} z_{j}\right) \approx$ $\sum_{j=1, \ldots, J} \exp \left(-\frac{z_{j}}{\gamma}\right)$. This method is simple and computationally efficient. This approximation is also known as the LogSumExp function when $\gamma<0$.

Smooth-min approximation [10]: This approach introduces parameters $\beta, Z>0$ and approximates the minimum as: $\min _{j=1, \ldots, J} z_{j} \approx \frac{\sum_{j=1}^{J} z_{j} \exp \left(-\beta z_{j}\right)}{Z}$. Given properly chosen parameters, this approximation error can be arbitrarily small. This approach is equivalent to a smooth-max function when $\beta<0$.

\section{FRÉCHET KERNEL (FRK)}

We develop the Fréchet kernel (FRK) by approximating non-smooth operations of the Fréchet distance using smooth approximation techniques. We rigorously resolve the non-smoothness of the min operation for alignments and the max operation for the pairs of indices in (1). This section describes each of these steps.

(i) Soft-min approximation for alignments: We approximate the min operation $\min _{A \in A}$ on the alignments in (1) using the soft-min approximation with $\gamma>0$ as: $\exp \left(-D_{\text {Frchet }}(X, Y)\right) \approx \sum_{A \in \mathcal{A}} \min _{(i, j) \in A} \phi\left(x_{i}, y_{j}\right)$. where we define $\phi\left(x_{i}, y_{j}\right)=\exp \left(-d\left(x_{i}, y_{j}\right) / \gamma\right)$.

(ii) Smooth-min approximation for location pairs: We approximate the remaining min operation using the smooth-min approximation. With parameters $\beta>0$ and $Z_{A}>0$ for each $A \in \mathcal{A}$, we obtain: $\min _{(i, j) \in A} \phi\left(x_{i}, y_{j}\right) \approx \sum_{(i, j) \in A} \frac{\phi\left(x_{i}, y_{j}\right) \exp \left(-\beta \phi\left(x_{i}, y_{j}\right)\right)}{Z_{A}}$.

(iii) Fréchet Kernel: We develop our proposed Fréchet kernel by combining (i) and (ii) as :

$$
k_{\mathrm{F}}(X, Y):=\sum_{A \in \mathcal{A}} \sum_{(i, j) \in A} \frac{\widetilde{\phi}_{\varepsilon}\left(x_{i}, y_{j}\right) \exp \left(-\beta \phi\left(x_{i}, y_{j}\right)\right)}{Z_{A}},
$$

where $\widetilde{\phi}_{\varepsilon}\left(x_{i}, y_{j}\right):=\exp \left(-d\left(x_{i}, y_{j}\right) / \gamma+\varepsilon \delta\left(x_{i}-y_{j}\right)\right)$ with a parameter $\varepsilon>0$. Here, $\delta(t)$ is the delta function which returns 1 if $t=0$ and returns 0 otherwise. We add this term for regularization.

\subsection{Positive Definite Property}

In preparation, we define a formal sample space. Set $M=\max \{p, q\}$ and $\mathcal{X} \subset \mathbb{R}^{D}$ as a space of each element $x_{i}$ and $y_{j}$ from $X=$ $\left(x_{1}, x_{2}, \ldots x_{p}\right)$ and $Y=\left(y_{1}, y_{2}, \ldots, y_{q}\right)$. Then, we define the sample space as $\mathcal{X}^{*}=\sup _{j=1}^{M} \mathcal{X}^{\otimes j}$ and regard $k_{\mathrm{F}}$ as a kernel function on $X^{*} \times X^{*}$. We also consider a symmetric property of alignments: for any alignment $A$, its symmetric object is defined as $\widetilde{A}:=\{(j, i) \mid$ $(i, j) \in A\}$.

We now discuss the positive definite property of FRK, that is, for any $n$ pairs $\left\{\left(w_{i}, X_{i}\right)\right\}_{i=1}^{n} \subset \mathbb{R} \times \mathcal{X}^{*}, \sum_{i, j=1}^{n} w_{i} w_{j} k_{\mathrm{F}}\left(X_{i}, X_{j}\right) \geq 0$ holds. This is a fundamental property of the kernel method and is quite significant for its usage in ensuring as consistent prediction and desirable distribution embedding. The result is provided by the following theorem.

Theorem 1. For $x, y \in X$, set $d(x, y)=\|x-y\|_{2}^{2}$. Then, $k_{\mathrm{F}}:$ $\mathcal{X}^{*} \times \mathcal{X}^{*} \rightarrow \mathbb{R}$ is a positive definite kernel function for any $\gamma, \varepsilon>0$ and $\beta \leq \bar{\beta}$ with an existing threshold $\bar{\beta}>0$.

Proof Sketch: First, we show the positive definite properties of the internal functions that take each $\left(x_{i}, y_{j}\right)$ as an input. We obtain the result by using the delta function for regularization and the Schoenberg's theorem [11], which guarantees the positive definite property through the exponential function. Second, we show the positive definite property of a kernel function on $X^{*} \times X^{*}$. This is done by following the procedures outlined in [6], which makes the kernel to generate a block-wise positive definite Gram matrix. Third, we show the conclusion of Theorem 1 . This is obtained from 
the fact that a non-negative sum or expectation of kernels maintains its positive definite properties.

\section{ALGORITHM}

We develop computational algorithms to compute $k_{\mathrm{F}}$ in (2). A naive calculation requires exponential time because the number of all possible alignments increases exponentially with the length of trajectories. A typical DP approach with convolution kernels is thus not applicable for $k_{\mathrm{F}}$ when we set $Z_{A}=\sum_{(i, j) \in A} \exp \left(-\beta \phi\left(x_{i}, y_{j}\right)\right)$.

As preparation for our computational algorithm, we propose a probabilistic approximation for $k_{\mathrm{F}}$. We generalize the outer summation in (2) by an expectation in terms of $A \in \mathcal{A}$ associated with a probabilistic function $P(A)$. Then, we define an expectation of $k_{\mathrm{F}}(X, Y)$ over alignments:

$$
\widehat{k}_{F}(X, Y):=\mathbb{E}_{A \sim P}\left[\sum_{(i, j) \in A} \frac{\widetilde{\phi}_{\varepsilon}\left(x_{i}, y_{j}\right) \exp \left(-\beta \phi\left(x_{i}, y_{j}\right)\right)}{Z_{A}}\right]
$$

We note that $\widehat{k}_{F}$ is also a positive definite kernel on $\mathcal{X}^{*} \times \mathcal{X}^{*}$, when $P(A)$ is symmetric for alignments, that is, $P(A)=P(\widetilde{A})$ holds for any $A \in \mathcal{A}$.

We also prepare a non-negative integer matrix $\mathcal{N} \in \mathbb{N} p \times q$ as a precomputation. Its $(i, j)$-th element $\mathcal{N}_{i, j}$ represents the number of all possible alignments for two trajectories of lengths $i$ and $j . \mathcal{N}$ is precomputed just once by the DP scheme as follows. We initialize a part of the elements as $\mathcal{N}_{i, 0}=\mathcal{N}_{0, j}=0, i=1, \ldots, p, j=1, \ldots, q$, then calculate the other elements as:

$$
\mathcal{N}_{i, j}= \begin{cases}1 & \text { if } i=1 \vee j=1 \\ \mathcal{N}_{i-1, j}+\mathcal{N}_{i, j-1}+\mathcal{N}_{i-1, j-1} & \text { otherwise. }\end{cases}
$$

It requires $O(p q)$ computational time.

We present an efficient algorithm which consists of sampling a set of alignments from a probability function $P(A)$. FRK considers $P(A)$ as a uniform distribution on $\mathcal{A}$ and generates a random sampling of $A$. It then takes the following three steps. (i) We first set $(p, q)$ as an initial pair of indices. (ii) From a current index pair $(i, j)$, we move from $(i, j)$ to one of three pairs $(i-1, j),(i, j-1)$, or $(i-1, j-1)$ with probabilities of $\mathcal{N}_{i-1, j} / \mathcal{N}_{i, j}, \mathcal{N}_{i, j-1} / \mathcal{N}_{i, j}$, and $\mathcal{N}_{i-1, j-1} / \mathcal{N}_{i, j}$, respectively. (iii) Repeat step (ii) until we reach $(1,1)$. The randomly sampled pass from $(p, q)$ to $(1,1)$ corresponds to a set of sampled alignments. When we repeat the steps $K$ times, $K$ alignments are randomly sampled. The time complexity for sampling $K$ alignments is $O(K(p+q))$ because the maximum length of an alignment is $p+q-1$. Our algorithm runs in $O(K(p+q))$ time.

\section{RELATED WORK}

The Fréchet distance [9] and its variants [1] have been applied in a wide range of trajectory analysis problems, such as similarity search [26], and clustering [4]. Although the kernel leveraging benefits of the Fréchet distance is typically expected to be useful in trajectory data analysis, no prior work on kernels for the Fréchet distance has been conducted to validate the expectation. In time series analysis, various measures based on DTW have been proposed for evaluating the similarity between time series [22]. The move-split-merge metric (MSM) [27] and the time warp edit distance (TWE) [18] have been proposed to elastically measure the similarity by using

\begin{tabular}{|c|c|c|c|c|c|c|}
\hline Methods & ABC2018 & Geolife & NBA & Pedestrian & Cyclist & Ave. \\
\hline FRK & $\underline{0.73}(0.05)$ & $0.94_{(0.01)}$ & $\underline{0.74}(0.02)$ & $\underline{0.99}(0.01)$ & $\underline{0.99}(0.01)$ & $\underline{0.88}$ \\
\hline Fréchet & $0.65_{(0.07)}$ & $0.94_{(0.01)}$ & $0.608_{(0.03)}$ & $0.97_{(0.02)}$ & $0.98_{(0.02)}$ & 0.83 \\
\hline Hausdorff & $0.67_{(0.08)}$ & $0.94_{(0.01)}$ & $0.67_{(0.03)}$ & $0.95_{(0.02)}$ & $0.97(0.04)$ & 0.84 \\
\hline DTW & $0.60_{(0.05)}$ & $0.83_{(0.01)}$ & $0.56_{(0.03)}$ & $0.89_{(0.03)}$ & $0.93_{(0.04)}$ & 0.76 \\
\hline MSM & $0.67_{(0.06)}$ & $\underline{0.95}(0.01)$ & $0.70_{(0.02)}$ & $0.95_{(0.02)}$ & $0.98_{(0.02)}$ & 0.85 \\
\hline TWED & $0.63_{(0.07)}$ & $0.93_{(0.01)}$ & $0.71_{(0.02)}$ & $0.87_{(0.03)}$ & $0.92_{(0.02)}$ & 0.81 \\
\hline KDTW & $0.49_{(0.09)}$ & $0.63_{(0.04)}$ & $0.50_{(0.01)}$ & $0.66_{(0.10)}$ & $0.68_{(0.09)}$ & 0.59 \\
\hline GA & $0.51_{(0.01)}$ & $0.50_{(0.01)}$ & $0.50_{(0.01)}$ & $0.50_{(0.01)}$ & $0.51_{(0.01)}$ & 0.51 \\
\hline LSTM & $0.62_{(0.06)}$ & $0.88_{(0.02)}$ & $0.72_{(0.02)}$ & $0.84_{(0.06)}$ & $0.88_{(0.05)}$ & 0.79 \\
\hline GRU & $0.62_{(0.07)}$ & $0.91_{(0.01)}$ & $0.73_{(0.02)}$ & $0.95_{(0.02)}$ & $0.93_{(0.05)}$ & 0.83 \\
\hline Traj2vec & $0.51_{(0.07)}$ & $0.49_{(0.05)}$ & $0.47_{(0.02)}$ & $0.49_{(0.03)}$ & $0.47_{(0.07)}$ & 0.49 \\
\hline
\end{tabular}

Table 1: Average (standard deviation) of the test AUC scores.

the edit-distance and combining LCSS and DTW, respectively. Dynamic time warping kernel (KDTW) [19] is a regularized version of DTW. Recently, yet another approach employing neural networks for learning representations from trajectories has attracted considerable interest. [31] proposed a trajectory clustering method using an RNN and achieved significant improvements compared to other metrics, such as DTW and the Hausdorff distances. [15] and [30] proposed similar methods using RNNs for similarity search tasks.

\section{EXPERIMENT}

We conducted intensive trajectory classification experiments with five publicly available trajectory data sets. ABC2018 ${ }^{1}$ dataset, which contains GPS trajectories $(N=906)$ of seabirds (Calonectris leucomelas), recorded over six years. Because their behavior is known to vary by sex [20], we employed Sex $=\{$ Male, Female $\}$ as labels. Geolife [33] dataset, which contains the GPS trajectories of users in Beijing. We extracted $N=3278$ trajectories recorded from users riding a bus, bike, car, or taxi. NBA dataset, which contains the tracking trajectories for professional basketball players $(N=3000)$ recorded by the SportVU system. We employed the three positions of players \{Guard, Forward, Center\} as labels. The dataset was randomly sampled from the NBA 2015-2016 season ${ }^{2}$. VRU (pedestrian) ${ }^{3}$ dataset, which contains pedestrian trajectories recorded at an urban intersection. For this dataset, we used three types of labels: moving, starting, and stopping. VRU (cyclist) dataset, which contains cyclist trajectories.

To classify trajectories, we employed an SVM [28] with our FRK. As baselines, we utilized an SVM using a pseudo-kernel with the Fréchet distance (Fréchet), the Hausdorff distance (Hausdorff) [13], Dynamic Time Warp distance (DTW), the Move-Split-Merge Metric (MSM) [27], the Time Warp Edit Distance (TWED) [18], and kernels including Dynamic Time Warping Kernel (KDTW) [19], Global alignment (GA) kernel. We used the RBF kernel $k(X, Y)=$ $\exp \left(-\frac{1}{2 \gamma} D(X, Y)\right)$ to construct the pseudo-kernels where $D(X, Y)$ represents each corresponding distance. We utilized the local kernel for GA by following [5]. As another state-of-the-art baseline comparison, we employed two bidirectional RNNs using the long

\footnotetext{
${ }^{1}$ https://competitions.codalab.org/competitions/16283

${ }^{2}$ https://www.stats.com/data-science/

${ }^{3}$ https://www.th-ab.de/vru-trajectory-dataset
} 
short-term memory (LSTM) and the gated recurrent units (GRU) for feature extraction from the trajectories, and a fully connected layer for predicting the label of trajectories. We also employed a logistic regression whose input features were the embeddings learned by [31] (Traj2vec).

We ran the experiments ten times, in each of which we randomly chose $90 \%$ of the trajectories as training data and the remaining as test data. We scaled the observed latitude and longitudes to $[0,1]$. Using five-fold cross validation, hyper-parameters of the kernels and pseudo-kernels $\gamma, \beta$, and $L$ were selected from $\left\{1.0,10^{-1}, \ldots, 10^{-8}\right\},\{10.0,1.0,0.1\}$ and $\{1,3,5\}$, respectively. For FRK, we set the hyper-parameter $K=100$. Since $\varepsilon>0$ in (2) is a theoretical value and does not affect the actual algorithm, its value was not chosen. We measured the test performance with the area under the curve (AUC) of the receiver operating characteristic curve for the binary label cases and their weighted scores for multi-label cases. For LSTM and GRU, we implemented models in Python and selected the number of hidden units from $\{32,64,128\}$ with the cross-entropy loss. For Traj2vec, we used the original code provided by authors to learn the trajectory embeddings. Our source code is available on our site ${ }^{4}$.

Table 1 shows the average and standard deviation of the test AUC. The best and second-best average scores are indicated in underline and italic type for each experiment. Our proposed methods FRK achieved significant improvements over the existing methods using SVM and the RNNs on the ABC2018, NBA, VRU (Pedestrian), and VRU (Cyclist) datasets. Because these datasets are noisier than the Geolife dataset, these results indicate that the smoothness and positive definiteness of FRK successfully extracted features by correctly measuring the similarity of noisy trajectories, which contributed to achieving high classification accuracies. Different properties of the datasets can affect the different performances of the two sampling methods, and can also vary the importance of the order of points in a trajectory.

\section{CONCLUSION}

We proposed a Fréchet kernel (FRK), which is applicable to commonly-used kernel methods. We integrated two smoothing approximation methods, the soft-min for alignments and smooth-min approximations for matched pairs, with the original Fréchet distance and developed a computationally efficient algorithm that can calculate our FRK for long trajectories. We conducted trajectory classification experiments and demonstrated the performance improvements of FRK from baselines.

\section{REFERENCES}

[1] Helmut Alt and Michael Godau. 1995. Computing the Fréchet Distance between Two Polygonal Curves. International fournal of Computational Geometry \& Applications 5 (03 1995), 75-91.

[2] Donald J. Berndt and James Clifford. 1994. Using Dynamic Time Warping to Find Patterns in Time Series. In Proceedings of AAAI workshop.

[3] Karl Bringmann. 2014. Why Walking the Dog Takes Time: Fréchet Distance Has No Strongly Subquadratic Algorithms Unless SETH Fails. In Proceedings of FOCS.

[4] Kevin Buchin, Anne Driemel, Joachim Gudmundsson, Michael Horton, Irina Kostitsyna, Maarten Löffler, and Martijn Struijs. 2019. Approximating $(k, \ell)$ center clustering for curves. In Proceedings of SODA.

[5] Marco Cuturi. 2011. Fast Global Alignment Kernels. In Proceedings of ICML.
[6] Marco Cuturi, Jean-Philippe Vert, Oystein Birkenes, and Tomoko aatsui. 2007. A Kernel for Time Series Based on Global Alignments. In Proceedings of ICASSP.

[7] Wenhao Ding, Mengdi Xu, and Ding Zhao. 2020. CMTS: A Conditional Multiple Trajectory Synthesizer for Generating Safety-Critical Driving Scenarios. In Proceedings of ICRA.

[8] Thomas Eiter and Heikki Mannila. 1994. Computing Discrete Fréchet Distance. Technical Report. Technical Report CD-TR 94/64, Christian Doppler Laboratory for Expert Systems, TU Vienna, Austria.

[9] M Maurice Fréchet. 1906. Sur quelques points du calcul fonctionnel. Rendiconti del Circolo Matematico di Palermo 22, 1 (1906), 1-72.

[10] Ian Goodfellow, Yoshua Bengio, Aaron Courville, and Yoshua Bengio. 2016. Deep Learning. MIT Press.

[11] J. C Guella and Valdir Antônio Menegatto. 2019. Schoenberg's Theorem for Positive Definite Functions on Products: A Unifying Framework. Fournal of Fourier Analysis and Applications 25, 4 (2019), 1424-1446.

[12] Agrim Gupta, Justin Johnson, Li Fei-Fei, Silvio Savarese, and Alexandre Alahi. 2018. Social GAN: Socially Acceptable Trajectories With Generative Adversarial Networks. In Proceedings of CVPR.

[13] Daniel P. Huttenlocher, Gregory A. Klanderman, and William A. Rucklidge. 1993. Comparing Images Using the Hausdorff Distance. IEEE Transactions on Pattern Analysis and Machine Intelligence 15, 9 (1993), 850-863.

[14] John Krumm and Eric Horvitz. 2004. LOCADIO: Inferring Motion and Location from Wi-Fi Signal Strengths.. In Proceedings of MOBIQUITOUS.

[15] Xiucheng Li, Kaiqi Zhao, Gao Cong, Christian S Jensen, and Wei Wei. 2018. Deep Representation Learning for Trajectory Similarity Computation. In Proceedings of ICDE.

[16] Junwei Liang, Lu Jiang, Juan Carlos Niebles, Alexander G. Hauptmann, and Li Fei-Fei. 2019. Peeking Into the Future: Predicting Future Person Activities and Locations in Videos. In Proceedings of CVPR.

[17] Yuexin Ma, Xinge Zhu, Sibo Zhang, Ruigang Yang, Wenping Wang, and Dinesh Manocha. 2019. Trafficpredict: Trajectory prediction for heterogeneous trafficagents. In Proceedings of AAAI.

[18] Pierre-François Marteau. 2009. Time Warp Edit Distance with Stiffness Adjustment for Time Series Matching. IEEE Transactions on Pattern Analysis and Machine Intelligence 31, 2 (2009), 306-318.

[19] Pierre-François Marteau and Sylvie Gibet. 2015. On Recursive Edit Distance Kernels With Application to Time Series Classification. IEEE Transactions on Neural Networks and Learning Systems 26, 6 (2015), 1121-1133.

[20] Sakiko Matsumoto, Takashi Yamamoto, Maki Yamamoto, Carlos B Zavalaga, and Ken Yoda. 2017. Sex-related differences in the foraging movement of streaked shearwaters Calonectris leucomelas breeding on Awashima Island in the Sea of Japan. Ornithological Science 16, 1 (2017), 23-32.

[21] Jonathan Owens and Andrew Hunter. 2000. Application of the self-organising map to trajectory classification. In Proceedings of VS-PETS workshop.

[22] John Paparrizos, Chunwei Liu, Aaron J. Elmore, and Michael J. Franklin. 2020. Debunking Four Long-Standing Misconceptions of Time-Series Distance Measures. In Proceedings of SIGMOD.

[23] Donald J Patterson, Lin Liao, Dieter Fox, and Henry Kautz. 2003. Inferring High-Level Behavior from Low-Level Sensors. In Proceedings of UbiComp.

[24] Sean L. Seyler, Avishek Kumar, M. F. Thorpe, and Oliver Beckstein. 2015. Path Similarity Analysis: A Method for Quantifying Macromolecular Pathways. PLOS Computational Biology 11 (2015), 1-37.

[25] Timothy Sohn, Alex Varshavsky, Anthony LaMarca, Mike Y. Chen, Tanzeem Choudhury, Ian Smith, Sunny Consolvo, Jeffrey Hightower, William G. Griswold, and Eyal de Lara. 2006. Mobility Detection Using Everyday GSM Traces. In Proceedings of UbiComp.

[26] E. Sriraghavendra, K. Karthik, and C. Bhattacharyya. 2007. Fréchet Distance based Approach for Searching Online Handwritten Documents. In Proceedings of ICDAR.

[27] Alexandra Stefan, Vassilis Athitsos, and Gautam Das. 2013. The Move-Split-Merge Metric for Time Series. IEEE Transactions on Knowledge and Data Engineering 25, 6 (2013), 1425-1438.

[28] Ingo Steinwart and Andreas Christmann. 2008. Support vector machines. Springer Science \& Business Media.

[29] Tong Liu, P. Bahl, and I. Chlamtac. 1998. Mobility modeling, location tracking, and trajectory prediction in wireless ATM networks. IEEE fournal on Selected Areas in Communications 16, 6 (1998), 922-936. https://doi.org/10.1109/49.709453

[30] Zheng Wang, Cheng Long, Gao Cong, and Ce Ju. 2019. Effective and Efficient Sports Play Retrieval with Deep Representation Learning. In Proceedings of SIGKDD.

[31] Di Yao, Chao Zhang, Zhihua Zhu, Jianhui Huang, and Jingping Bi. 2017. Trajectory Clustering via Deep Representation Learning. In Proceedings of IfCNN.

[32] Yu Zheng. 2015. Trajectory Data Mining: An Overview. ACM Transactions on Intelligent Systems and Technology 6, 3 (2015).

[33] Yu Zheng, Quannan Li, Yukun Chen, Xing Xie, and Wei-Ying Ma. 2008. Understanding Mobility Based on GPS Data. In Proceedings of UbiComp.

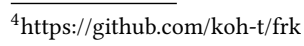

